\title{
RECOBA: Um ambiente para publicação e compartilhamento de Objetos de Aprendizagem no formato aberto e fragmentado
}

\author{
Rossana de Paula Junqueira ${ }^{1,2}$, Bernadette Farias Lóscio ${ }^{1}$ \\ ${ }^{1}$ Centro de Informática - Universidade Federal de Pernambuco (UFPE) \\ Caixa Postal 7851 - 50.732-970 - Recife - PE - Brasil \\ ${ }^{2}$ Instituto Federal do Sertão Pernambucano (IFSertão-PE) - Campus Petrolina \\ BR 407, KM 08 - Jardim São Paulo - 56.314-520 - Petrolina - PE - Brasil \\ rpj@cin.ufpe.br, bfl@cin.ufpe.br
}

\begin{abstract}
Resumo. Para garantir a sua reutilização, um Objeto de Aprendizagem (OA) deve ser livremente utilizado, reutilizado e redistribuído por qualquer um. Para promover o reúso dos OAs, eles precisam ser compartilhados em formatos abertos, ou seja, formatos estruturados e legíveis por máquinas, e que possam ser novamente usados de forma livre pela sociedade. Outro fator que contribuiria para o seu reúso seria disponibilizá-lo de forma fragmentada, ou seja, dividido em pequenas partes. Uma vez criados os OAs, espera-se que eles sejam armazenados e disponibilizados em um local adequado, como os Repositórios de Objetos de Aprendizagem. Este trabalho propõe a criação de um ambiente para publicação e compartilhamento de Objetos de Aprendizagem, utilizando formatos de dados abertos, e ainda sugerindo a fragmentação dos objetos, ou seja, a publicação do objeto em partes e não apenas como um todo. $O$ intuito é estimular a reutilização de Objetos de Aprendizagem, contribuindo, dessa forma, para o fortalecimento da aprendizagem, disseminação e aquisição de novos conhecimentos.
\end{abstract}

\begin{abstract}
To ensure its reuse, a Learning Object (LO) must be freely used, reused and redistributed by anyone. To promote the reuse of LOs, they need to be shared in open formats, ie, structured and machine-readable formats, and can be re-used freely by society. Another factor that would contribute to their reuse would make it available in a fragmented way, ie, divided into small parts. Once created the LOs, it is expected that they will be stored and made available in a suitable place, as the repositories of learning objects. This paper proposes the creation of an environment for publishing and sharing learning objects, using open data formats, and even suggesting the fragmentation of objects, ie, the publication of the object in parts and not just as a whole. The aim is to encourage reuse of learning objects, and thereby contributes to the strengthening of learning, dissemination and acquisition of new knowledge.
\end{abstract}

\section{INTRODUÇÃO}

Os constantes avanços tecnológicos nas áreas da informação e comunicação têm provocado profundas mudanças na sociedade. A facilidade de compartilhamento de 


\section{CBIE-LACLO 2015}

Anais dos Workshops do IV Congresso Brasileiro de Informática na Educação (CBIE 2015)

informação, favorecida por estas tecnologias, tem contribuído para a Web se tornar um imenso repositório de conteúdos, de tamanho considerável em diversos domínios do conhecimento.

De maneira semelhante, a educação aberta e a educação à distância aparecem, cada vez mais, no contexto das sociedades contemporâneas, como uma modalidade de educação que favorece a flexibilidade de horários e localização aos aprendizes que não podem estar fisicamente presentes em um ambiente presencial de ensino-aprendizagem. Neste contexto, surgem novas soluções, como as baseadas em Objetos de Aprendizagem (OAs), com o intuito de facilitar ainda mais o compartilhamento de conteúdos educacionais disponíveis na Web [Willey 2000].

Apesar de ser um termo bastante difundido, ainda não há um consenso na literatura para a definição de um OA. Os conceitos tendem para a ideia de que um OA deve possuir um conteúdo educacional pronto para ser utilizado em vários cenários e contextos de aprendizagem [Willey 2000]. Podemos citar como exemplos de OAs: vídeos, imagens, hipertextos, apresentação de slides, livros e apostilas [Dias et al. 2009].

Após os OAs serem criados, os mesmos são disponibilizados para uso de alunos e/ou professores, e devem possuir um caráter de reusabilidade, ou seja, a possibilidade de ser reutilizado em vários contextos por usuários distintos. Contudo, considerando a grande quantidade de materiais existentes e o formato no qual estão disponibilizados, selecionar o conteúdo adequado a uma determinada situação de aprendizagem pode não ser uma tarefa simples. Para facilitar o acesso aos OAs, existem os repositórios de OAs que são locais apropriados para armazenamento e disponibilização de objetos de aprendizagem [Rocha et al. 2011]. Porém, em geral, os OAs não são disponibilizados em um formato adequado que facilite seu compartilhamento e reúso por grupos distintos de usuários.

Uma forma de facilitar o compartilhamento e reúso de OAs consiste em disponibilizá-los em formato aberto, i.e. estruturado e legível por máquinas, a fim de que possam ser novamente usados de forma livre por qualquer um que tenha interesse em seu conteúdo. Estando dessa forma, os OAs se encaixam no conceito de Dado Aberto (Open Data). Segundo a organização Open Knowledge Foundation ${ }^{1}$, Dados Abertos são dados que podem ser livremente usados, reutilizados e redistribuídos por qualquer pessoa - sujeitos, no máximo, à exigência de atribuição da fonte e compartilhamento pelas mesmas regras.

Além da disponibilização em formato legível por máquina, a fim de facilitar o reúso dos OAs, é importante que este seja disponibilizado em formato não proprietário e de forma fragmentada, ou seja, ao invés de disponibilizar os OAs como um todo, devem ser disponibilizados fragmentos dos OAs [Amorim et al. 2006]. No caso do objeto de aprendizagem livro, por exemplo, a sua fragmentação poderia ser feita da seguinte forma: o livro possui diversos capítulos, cada capítulo possui várias seções, cada seção é dividida em vários parágrafos, figuras, tabelas e gráficos. Dessa forma, ao invés de disponibilizar apenas o livro como um todo, poderiam ser disponibilizados trechos do livro, ou seja, capítulos, seções ou até mesmo figuras.

\footnotetext{
${ }^{1}$ http://opendefinition.org/
} 
Para facilitar a representação de OAs na forma de fragmentos, modelos semânticos, como as ontologias, podem ser utilizados. Utilizar um modelo semântico e o conceito de dados abertos para disponibilizar fragmentos de objetos de aprendizagem contribui para o reúso de conteúdos educacionais, uma vez que facilita a produção de novos materiais educacionais a partir de objetos de aprendizagem existentes.

Neste contexto, este trabalho propõe a criação de um ambiente para publicação de OAs em formato aberto, denominado RECOBA, onde tecnologias semânticas, como as ontologias, são utilizadas para facilitar a fragmentação dos OAs. Especificamente, o ambiente proposto permite a publicação e o compartilhamento de OAs em formato aberto e fragmentado, de tal forma que usuários possam facilmente localizá-los e reutilizá-los, diferentemente da forma como os OAs encontram-se disponíveis nos repositórios atualmente disponíveis na Web.

O restante deste artigo está organizado da seguinte forma: a Seção 2 apresenta a metodologia utilizada no desenvolvimento do trabalho, a Seção 3 discute a criação do ambiente desenvolvido, a Seção 4 descreve a avaliação do ambiente, a Seção 5 fala sobre os Trabalhos Relacionados, que cita a análise comparativa que foi realizada nos repositórios encontrados na web, a fim de encontrar lacunas a serem solucionados no ambiente que está sendo proposto e a Seção 6 apresenta as considerações finais.

\section{METODOLOGIA}

Este trabalho foi realizado em várias etapas. Inicialmente, foi realizado um estudo bibliográfico envolvendo as principais áreas abordadas nesta pesquisa, entre elas: Objetos de Aprendizagem, Dados Abertos, Recursos Educacionais Abertos, Repositórios de Objetos de Aprendizagem e Ontologias.

Após o estudo bibliográfico inicial, foram analisados os repositórios de OAs já existentes com a finalidade de levantar as suas deficiências e, a partir delas, propor um novo ambiente com diferenciais em relação aos existentes. Como resultado da análise comparativa dos repositórios [Junqueira and Lóscio 2014], verificou-se, que algumas características dos OAs, dos Dados Abertos e dos REAs, que podem contribuir para a reusabilidade dos OAs ainda não eram atendidas pelos repositórios existentes, tais como, a disponibilização em um formato que facilite sua reutilização e a disponibilização de OAs de forma fragmentada.

Em seguida, fizemos a especificação do ambiente e implementamos o que foi especificado, criando um ambiente para publicação e compartilhamento de Objetos de Aprendizagem no formato aberto e fragmentando com o objetivo de promover o reúso dos objetos.

Por fim, foi realizada uma avaliação do ambiente implementado por meio de experimentos com a participação de usuários a fim de verificar se realmente o ambiente está promovendo o reúso dos Objetos de Aprendizagem.

\section{O AMBIENTE RECOBA}

Nesta seção, apresentamos uma visão geral do ambiente proposto neste trabalho, bem como descrevemos as principais atividades que envolvem o processo de publicação de um $\mathrm{OA}$ em formato aberto e fragmentado. 
CBIE-LACLO 2015

Anais dos Workshops do IV Congresso Brasileiro de Informática na Educação (CBIE 2015)

\subsection{Visão Geral do RECOBA}

A Figura 1 apresenta a arquitetura do ambiente proposto, o RECOBA ${ }^{2}$, com os seus principais componentes, os quais são descritos a seguir.

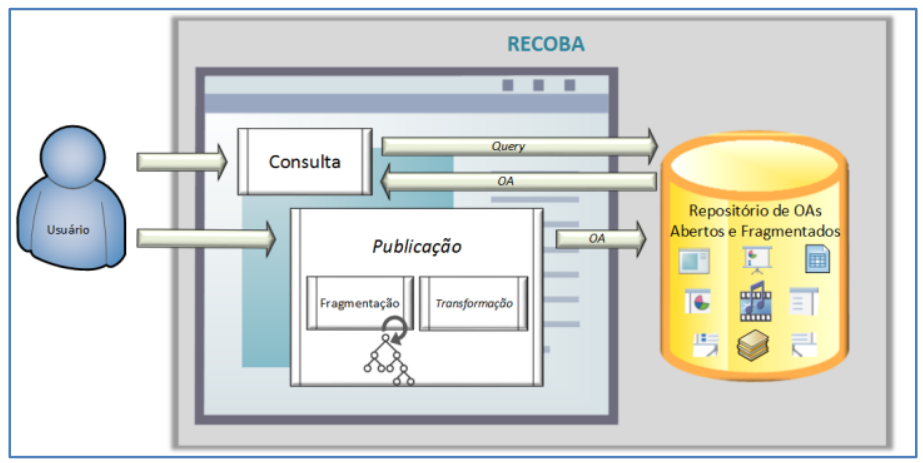

Figura 1: Arquitetura Proposta

Fonte: [Junqueira 2015]

O componente principal do ambiente RECOBA é o Repositório de OAs, onde ficam armazenados os OAs na sua integralidade (por exemplo, um livro) ou de forma fragmentada (por exemplo, uma figura que pertence a um livro). Ressaltando que esses OAs precisam estar armazenados em um formato que permitam sua reutilização.

O usuário, a partir de uma interface gráfica, poderá interagir com os módulos Consulta e Publicação. No módulo Consulta, o usuário pode inserir um termo para busca e receberá como resultado os OAs ou fragmentos de OAs que satisfazem a sua consulta. No módulo de Publicação, o usuário irá realizar a publicação de OAs no repositório. O módulo de Publicação possui dois componentes: o módulo de Fragmentação e o módulo de Transformação.

No módulo de Transformação, o objeto de aprendizagem de entrada sofrerá algumas transformações para facilitar a fragmentação e a disponibilização dos fragmentos em diferentes formatos. No módulo de Fragmentação, o componente de destaque é a ontologia que será usada como base para a publicação dos OAs. Neste trabalho, será utilizada como base para definir o tamanho do fragmento, a ontologia ONTOER+ [Nogueira et al. 2014]. A ONTOER+ é baseada em metadados e descrição de conteúdo, o que facilita o entendimento para se obter um nível de granularidade menor, uma das características de fragmentação dos REA. Pensando-se no reúso dos OAs, optamos escolhemos usar a ONTOER+ por ser a única ontologia que trata realmente da disponibilização de OAs de forma fragmentada e que foi criada e implementada.

É importante ressaltar que, apesar de existirem outros objetos de aprendizagem que não sejam do tipo publicação, a versão atual do ambiente realiza a fragmentação apenas de Publicações. Consideramos OAs do tipo Publicação qualquer OA que possa ser representado como um documento textual, como artigos, livros, apostilas, trabalhos de conclusão de curso.

Já que estamos falando em dados abertos, neste projeto será aceito apenas o formato OpenDocument (ou OpenDocument Format - ODF) como formato de entrada

\footnotetext{
${ }^{2}$ http://recoba.hol.es/
} 
dos OAs. Este formato constitui um padrão aberto para o armazenamento de documentos. Um padrão aberto deve ser entendido como uma especificação disponível a qualquer desenvolvimento, com o objetivo de garantir a longevidade do conteúdo do documento, a interoperabilidade entre aplicativos e a independência de fornecedores (OpenDocument Format ${ }^{3}$ ). Utilizaremos a extensão .ODT para salvar os documentos.

Dentre os diferenciais do ambiente RECOBA, destacamos: ambiente de livre acesso, a facilidade de inclusão de novos objetos, a garantia de uso e reúso de objetos de aprendizagem, a publicação de objetos em formato editável, o uso de padrões de metadados para descrição dos objetos publicados, a fragmentação de objetos de acordo com um modelo semântico. Finalmente, o ambiente RECOBA é uma ferramenta livre, ou seja, o usuário do RECOBA tem a liberdade de executar o programa como desejar, para qualquer propósito. Seu o código está disponível no Github ${ }^{4}$ para dar liberdade de ser estudado como o programa funciona, e adaptá-lo às suas necessidades. , e ainda, o usuário tem a liberdade de distribuir cópias de suas versões modificadas a outros.

\subsection{Transformação dos Objetos de Aprendizagem}

Para gerar a fragmentação dos OAs precisaremos manipular o conteúdo do documento que representa os OAs. Através dos estudos realizados para este trabalho, foi possível constatar que o formato OpenDocument (ou OpenDocument Format - ODF) permitiria esta manipulação, além de constituir um padrão aberto para o armazenamento de documentos. Porém, para fazer a manipulação do documento original é preciso transformá-lo para um outro formato que permite acessar individualmente as diversas partes que compõem o documento, como parágrafos, tabelas, figuras, dentre outros.

A representação mais comum de um arquivo OpenDocument é por meio de uma coleção de subdocumentos dentro de um pacote compactado no formato ZIP. A estrutura de subdocumentos e diretórios de um arquivo OpenDocument de documento de texto apresenta uma separação significativa entre conteúdo e apresentação visual, conforme descrito a seguir:

- O diretório media guarda todas as imagens extraídas do documento principal.

- O diretório META-INF guarda um arquivo XML que apresenta as informações sobre o conteúdo do "pacote" OpenDocument.

- O arquivo content.xml armazena todo o conteúdo de texto do documento.

- O arquivo meta.xml contém os metadados do arquivo.

- O arquivo mimetype armazena a definição do tipo MIME do arquivo, seu objetivo é permitir a identificação do tipo de arquivo pelas aplicações e pelo sistema operacional.

- O arquivo settings.xml armazena as configurações de exibição como o fator de ampliação e a posição do cursor, ou seja, propriedades que não pertencem ao conteúdo ou à formatação.

- O arquivo styles.xml apresenta o conjunto de informações de formatação do documento, representado pelos estilos utilizados.

\footnotetext{
${ }^{3}$ http://www.opendocumentformat.org/

${ }^{4}$ https://github.com/prorecoba/fonte
} 


\section{CBIE-LACLO 2015}

Anais dos Workshops do IV Congresso Brasileiro de Informática na Educação (CBIE 2015)

Para fragmentar o objeto de aprendizagem, conforme proposto neste trabalho, utilizamos o diretório media que guarda todas as imagens inseridas no documento principal e o arquivo content.xml para gerar os vários fragmentos de texto.

\subsection{Fragmentação dos Objetos de Aprendizagem}

Nesta seção, descrevemos o processo de fragmentação dos objetos de aprendizagem do tipo Publicação. A fragmentação é feita a partir do arquivo content.xml obtido como resultado da descompactação dos OAs com a extensão ODT. Para cada um dos fragmentos obtidos a partir do documento principal, é gerado um arquivo xml, json e txt para ser disponibilizado ao usuário. Como nosso objetivo é disponibilizar dados em formato aberto, optamos por disponibilizar os fragmentos nas extensões xml e json pelo fato de serem formatos não proprietários, e que possibilitem o seu uso irrestrito e automatizado através da Web.

De maneira geral, o processo de fragmentação de um OA do tipo Publicação (Publication) consiste em percorrer o documento correspondente e criar fragmentos para cada um dos diversos componentes do documento, i.e., capítulos, seções, parágrafos, figuras, dentre outros. Em nossa abordagem, consideramos que um documento poderá ser dividido em vários Capítulos, que cada Capítulo pode ter várias Seções e/ou Blocos, e que cada Seção pode ter várias outras Seções e/ou Blocos. Consideramos ainda como Bloco, o menor fragmento que pode ser gerado, podendo ser um parágrafo ou uma imagem. Na versão atual do RECOBA, consideramos tabelas e quadros como sendo imagens.

O processo de fragmentação de um objeto de aprendizagem é guiado pela ontologia ONTOER+, ou seja, os fragmentos são criados de acordo com a hierarquia de classes proposta na ontologia, a qual é apresentada na Figura 2.

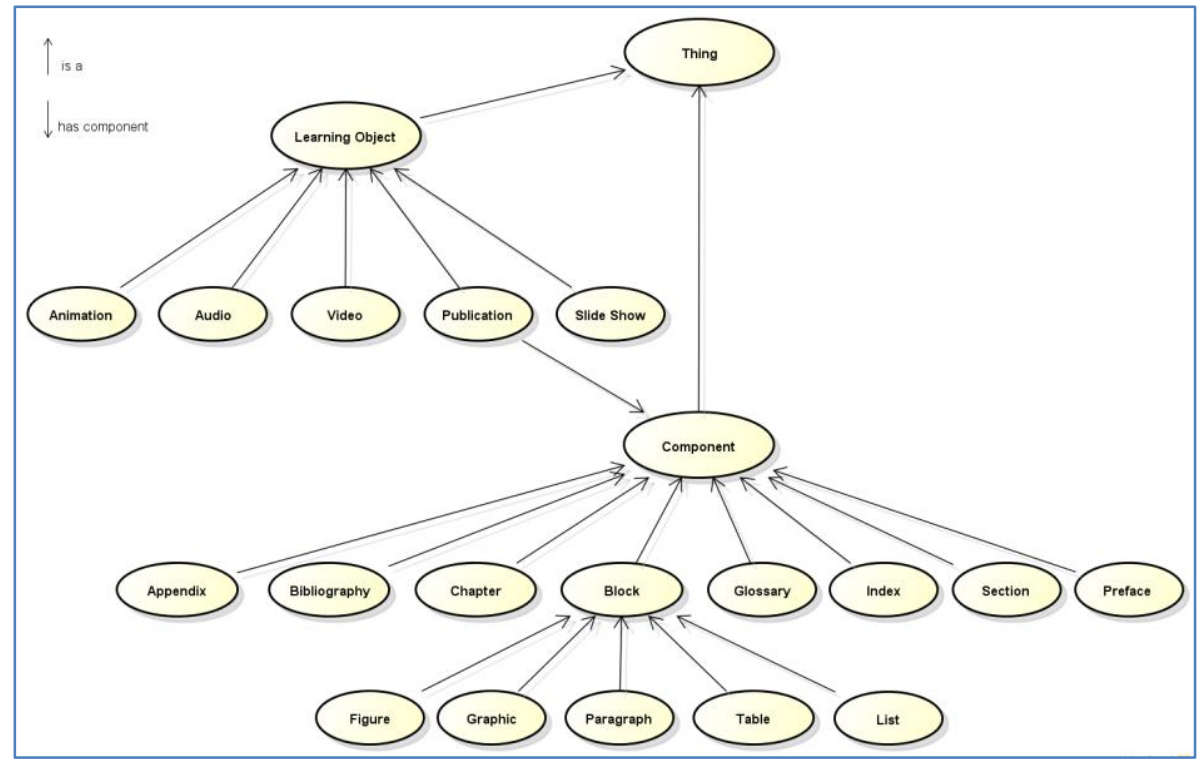

Figura 2: Hierarquia de classes ONTOER+.

Fonte: [Junqueira 2015]

Conforme podemos observar na Figura 2, Publication é uma subclasse de Learning Object. A classe Publication se relaciona com a classe Component por meio 
da propriedade de objeto has component. A classe Component, por sua vez, possui várias subclasses: Appendix, Bibliography, Chapter, Block, Glossary, Index, Section e Preface. De forma semelhante, Figure, Graphic, Paragraph, Table e List são subclasses de Block.

$\mathrm{Na}$ Figura 3 apresentamos de forma resumida a estrutura das tags do arquivo content.xml, gerado a partir de um arquivo de exemplo utilizado para fazer testes com o RECOBA. A fragmentação é feita a partir do arquivo content.xml obtido como resultado da descompactação dos OAs com a extensão ODT. Ao abrir o arquivo content.xml todo código é varrido a procura da tag $<$ text:h $>$. Esta tag identifica o início de um novo Título (Head). A tag <text:h> representa o início de novo título. O nível desta tag representado pelo text:outline-level especificará se neste ponto está iniciandose um novo Capítulo (text:outline-level=1) ou uma nova Seção dentro do Capítulo ou uma nova Seção dentro de uma outra Seção. (text:outline-level $>1$ ). Para cada tag $<$ text:p $>$ ou $<$ draw:image $>$ encontrada, consideramos como sendo um Bloco.

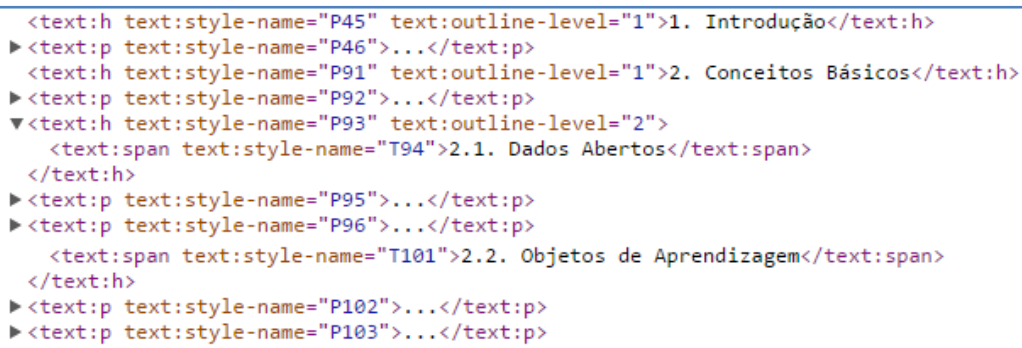

Figura 3: Tags resumidas do arquivo content.xml.

\section{Fonte: [Junqueira 2015]}

Nas figuras 4a, 4b e 4c ilustramos o resultado de um processo de fragmentação. $\mathrm{Na}$ Figura 4a são apresentados todos os fragmentos de Capítulos gerados pelo documento principal. Na Figura 4b, destacamos o capítulo de Introdução e apresentamos os fragmentos de Seções ou Parágrafos dentro do Capítulo destacado. E na Figura 4c, o destaque é para o texto de um Parágrafo.

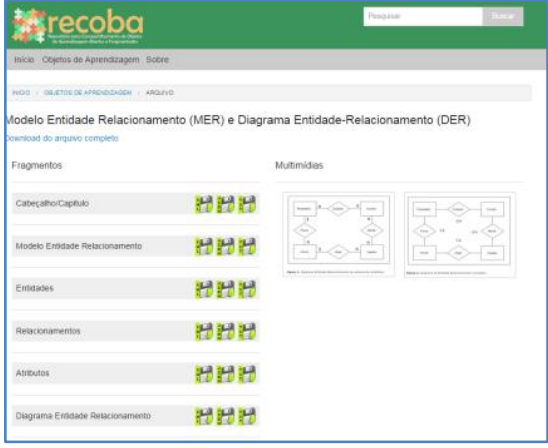

Figura 4a: Fragmentos do documento principal.

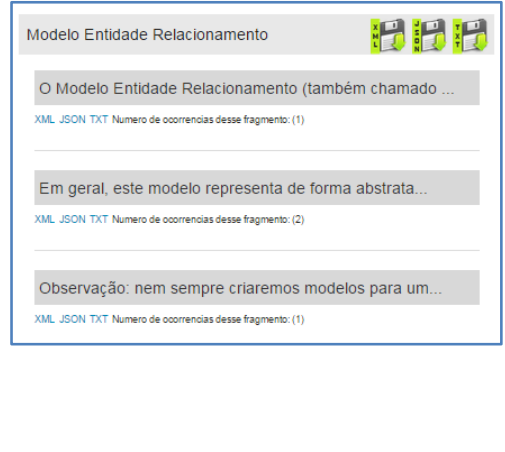

Figura 4b: Fragmentos de um capítulo.

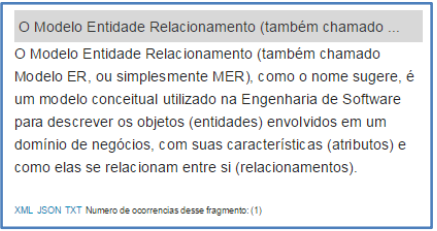

Figura 4c: Visualização de um parágrafo.

Fonte: Elaborado pela autora. 


\section{AVALIAÇÃO DO RECOBA}

O propósito do experimento foi verificar se a disponibilização do Objeto de Aprendizagem de forma fragmentada e em formato aberto promove o reúso dos OAs. Com o objetivo de verificar a quantidade de fragmentos que estão sendo reusados no momento da criação de novos OAs; verificar a efetividade com a qual o RECOBA realiza as atividades para as quais foi proposto; e, verificar a satisfação do usuário em utilizar o RECOBA.

Para avaliar o ambiente proposto, foi realizado um experimento com usuários, estudantes e/ou professores, de repositórios de OAs.

Para a realização dos testes, foi necessário preparar o RECOBA inserindo previamente alguns Objetos de Aprendizagem. O experimento foi realizado com 12 usuários, estudantes e/ou professores, que poderiam usar o RECOBA tanto para publicação quanto para consumo de OAs. Antes de iniciar o trabalho, foi feita uma breve apresentação do ambiente RECOBA para os presentes, esclarecendo o seu principal objetivo que é a disponibilização de OAs de forma fragmentada e em formato aberto.

As seguintes tarefas foram solicitadas aos usuários:

- A partir dos OAs já existentes no RECOBA, criar um novo OA do tipo Publicação;

- Incluir o novo OA criado no RECOBA e visualizar a porcentagem de fragmentos que teriam sido reusados;

Para a realização das tarefas os usuários podiam usar o RECOBA de forma livre, ou seja, podiam buscar por OAs livremente, para criar o seu novo OA. Depois de concluídas as tarefas, os usuários preencheram um questionário. O questionário foi dividido em três temas. O tema 1 busca verificar se o RECOBA realiza efetivamente as tarefas para as quais ele foi proposto. $\mathrm{O}$ tema 2 verifica o grau de satisfação do usuário ao utilizar o RECOBA. E o tema 3 verifica a proporção de fragmentos reutilizados.

A partir das respostas obtidas dos questionários, foram realizadas algumas análises, as quais são apresentadas a seguir.

Tema 1: Grau de efetividade das tarefas solicitadas ao RECOBA: Todos os usuários foram unânimes em afirmar que todas as tarefas solicitadas ao RECOBA foram concluídas efetivamente. Ou seja, o upload do Objeto de Aprendizagem, a visualização dos fragmentos gerados a partir do OA inserido, e a recuperação dos OAs e/ou seus fragmentos foram realizadas com sucesso.

Tema 2: Grau de satisfação dos usuários do RECOBA após utilizá-lo: Os resultados obtidos para as questões 4 (A forma como os fragmentos são apresentados é satisfatória), 5 (Os fragmentos gerados a partir do seu Objeto de Aprendizagem foram satisfatórios) e 6 (O resultado da pesquisa no RECOBA foi conforme esperado), coincidentemente foram os mesmos, conforme apresentado na Figura 5a. Sobre a interface do RECOBA, Questão 7, a avaliação dos usuários é apresentada na Figura 5b. 


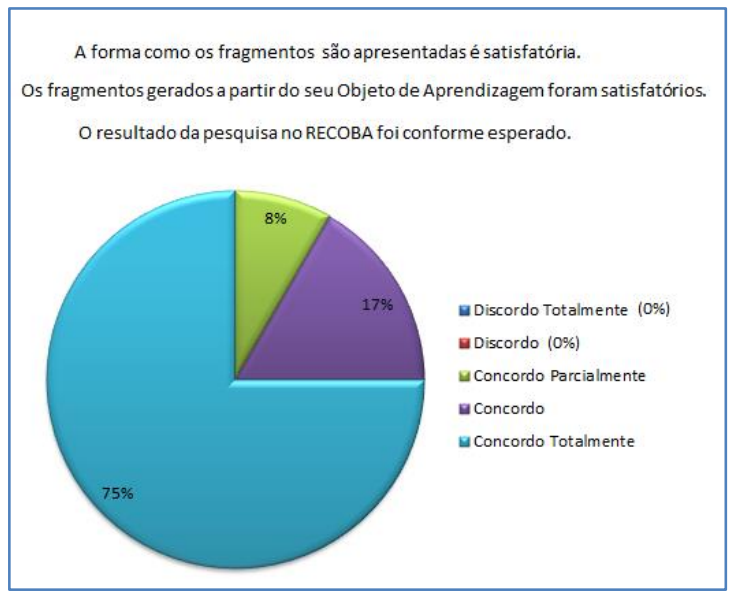

Figura 5a: Resultado das Questões 4, 5 e 6 do tema 2

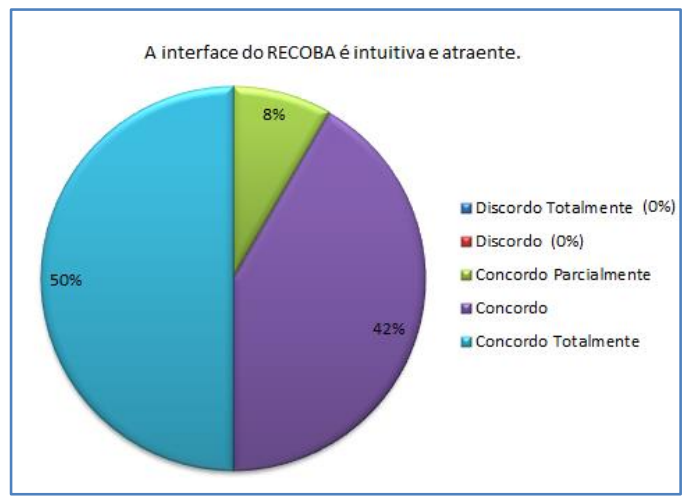

Figura 5b: Resultado da Questão 7 do tema 2

Fonte: [Junqueira 2015]

Tema 3: Medição do reúso de Objetos de Aprendizagem (OAs) com a disponibilização de OAs fragmentados: A média de reúso apresentada para os usuários foi de $41,27 \%$.

No geral, a partir das respostas dadas pelas pessoas que participaram do experimento do RECOBA e ainda com os valores do reúso de fragmentos de OAs gerados após a inclusão de um novo OA, podemos dizer que houve uma aprovação do ambiente, e que o mesmo promoveu o reúso dos Objetos de Aprendizagem.

Pôde-se perceber, que apesar de alguns autores relatarem que a reusabilidade de um OA não é uma tarefa trivial, podemos constatar que a partir do momento que se propõe a disponibilizar um OA em um formato que permite a sua edição e dividido em pequenas partes, ou seja, pequenos fragmentos, estaremos contribuindo para o seu reúso.

\section{TRABALHOS RELACIONADOS}

Para que pudéssemos propor um novo ambiente para publicação e compartilhamento de Objetos de Aprendizagem, fizemos uma análise comparativa de alguns dos principais repositórios de OAs atualmente disponíveis na Web [Junqueira and Lóscio 2014].

O objetivo da análise foi verificar se os conteúdos disponíveis nos repositórios poderiam ser classificados como OAs de fato e se poderiam ser usados e reusados em diferentes contextos. Após a seleção dos repositórios a serem considerados na comparação [Junqueira 2015], foi feita uma análise em cada repositório com a finalidade de avaliá-lo de acordo com as características dos Objetos de Aprendizagem: reusabilidade, interoperabilidade, granularidade, identificação por metadados, acessibilidade, agregação, autonomia, customização, digital, durabilidade, escalabilidades e facilidade para atualização. Foi apresentado o resultado da análise comparativa a fim de mostrar as lacunas existentes nos repositórios para que possamos propor um novo ambiente para disponibilização e compartilhamento de OAs [Junqueira 2015]. 


\section{CONCLUSÕES}

Este trabalho apresentou um novo ambiente, o RECOBA, para publicação e compartilhamento de Objetos de Aprendizagem em formato aberto e fragmentado. Esperamos com o RECOBA, promover o reúso dos OAs. Destacamos como principal contribuição deste trabalho o desenvolvimento de um ambiente que permite que qualquer usuário tenha livre acesso aos OAs disponibilizados, bem como possa incluir novos objetos de aprendizagem. Além disso, o ambiente proposto garante que todos os OAs disponibilizados podem ser usados e adaptados por terceiros, promovendo o uso e reúso de OAs. Também vale ressaltar que o RECOBA é uma ferramenta livre, ou seja, outras pessoas podem colaborar com melhorias para o RECOBA e podem fazer dos códigos que foram gerados.

\section{REFERÊNCIAS}

AMORIM, Ricardo R.; LAMA, Manuel; SÁNCHEZ, Eduardo; RIERA, Adolfo; VILA, Xosé A. A learning design ontology based on the IMS specification. In: Educational Technology \& Society, v. 9, n. 1, 2006. p. 38-57.

DIAS, Carla. C. L.; KEMCZINSKI, Avanilde; SÁ, Sérgio V.; FERLIN, Jhônatan; HOUNSELL, Marcelo S.. Padrões abertos: aplicabilidade em Objetos de Aprendizagem (OAs). In: Anais do XX Simpósio Brasileiro de Informática na Educação, SBIE, 2009.

JUNQUEIRA, Rossana de P.; LÓSCIO, Bernadette Farias. Repositórios de Objetos de Aprendizagem: uma análise comparativa com ênfase no reúso de conteúdos. In: Simpósio Brasileiro de Informática na Educação, SBIE, 2014.

JUNQUEIRA, Rossana de P. RECOBA: Um ambiente para publicação e compartilhamento de objetos de aprendizagem em formato aberto em fragmentado. Dissertação de Mestrado. Recife: Programa de Pós-Graduação em Computação do Centro de Informática da UFPE, 2015.

NOGUEIRA, Ubirajara S.; LOSCIO, Bernadette; AMORIM, Ricardo. Desenvolvimento de uma ontologia para recursos educacionais abertos com methontology. In: Simpósio Brasileiro de Informática na Educação, SBIE, 2014.

ROCHA, Farley; MORAES, Henrique; FABRI, Luciana; OLIVEIRA, Tárik; COSTA, Adriana; NETTO, Cristiane; COSTA, Helder; MORAIS, Rossana. Repositórios de objetos de aprendizagem - um estudo exploratório. In Simpósio Brasileiro de Informática na Educação, SBIE, 2011.

WILEY, D. A.. Learning object design and sequencing theory. 2000. Tese. In: $<$ http://www.reusability.org/read/chapters/wiley.doc $>$. Acessado em: $17 \mathrm{de}$ fev. 2014. 